\title{
A new approach to characterize very-low-level radioactive waste produced at hadron accelerators
}

\author{
Biagio Zaffora $^{\mathrm{a}, *}$, Matteo Magistris ${ }^{\mathrm{a}}$, Jean-Pierre Chevalier ${ }^{\mathrm{b}}$, Catherine Luccioni $^{\mathrm{b}}$, \\ Gilbert Saporta $^{\mathrm{b}}$, Luisa Ulrici ${ }^{\mathrm{a}}$ \\ a CERN, 1211 Geneva 23, Switzerland \\ b CNAM, 292 Rue Saint-Martin, 75003 Paris, France
}

\begin{abstract}
A B S T R A C T
Radioactive waste is produced as a consequence of preventive and corrective maintenance during the operation of high-energy particle accelerators or associated dismantling campaigns. Their radiological characterization must be performed to ensure an appropriate disposal in the disposal facilities. The radiological characterization of waste includes the establishment of the list of produced radionuclides, called "radionuclide inventory", and the estimation of their activity. The present paper describes the process adopted at CERN to characterize verylow-level radioactive waste with a focus on activated metals. The characterization method consists of measuring and estimating the activity of produced radionuclides either by experimental methods or statistical and numerical approaches. We adapted the so-called Scaling Factor (SF) and Correlation Factor (CF) techniques to the needs of hadron accelerators, and applied them to very-low-level metallic waste produced at CERN. For each type of metal we calculated the radionuclide inventory and identified the radionuclides that most contribute to hazard factors. The methodology proposed is of general validity, can be extended to other activated materials and can be used for the characterization of waste produced in particle accelerators and research centres, where the activation mechanisms are comparable to the ones occurring at CERN.
\end{abstract}

\section{Introduction}

The European Organization for Nuclear Research (CERN) operates a number of particle accelerators of high energy. Some beam particles can escape and interact with the accelerators' surroundings and structures, and therefore activate them. At the end of their lifetime, radioactive accelerator components and materials that cannot be reused need to be radiologically characterized prior to their disposal towards a disposal facility. The present study illustrates the process adopted at CERN to radiologically characterize very-low-level (French acronym: TFA - Très faible activité) waste which is eliminated towards the French disposal facility managed by ANDRA (French national radioactive waste management agency).

The chain leading to the final disposal of a batch of waste starts with the identification of the items to be disposed of. Items are included in a batch based on their similarity in terms of activation mechanisms, radiological history and chemical composition. Once a batch is identified and the relevant information collected (i.e. origin of the waste, irradiation history and chemical composition), we calculate the list of the produced radionuclides via either Monte Carlo simulations or analytical codes.

According to reference (IAEA, 2007), we classify each nuclide of the radionuclide inventory as follows:

- Easy-to-Measure (ETM) nuclides, such as $\gamma$-emitters, which can be measured via non-destructive nuclear assay means (e.g., in-situ $\gamma$ spectrometry)

- Difficult-to-Measure (DTM) nuclides, such as pure- $\beta$ emitters, which cannot be measured via non-destructive techniques (e.g., they require radiochemical analysis on samples)

- Impossible-to-Measure (ITM) nuclides, such as $\alpha$-emitters, pure- $\beta$ and low-energy X-emitters, which are very difficult to measure and which are, therefore, quantified via simulations or calculations.

From the list of ETMs we select the dominant $\gamma$-emitter, called Key Nuclide $(\mathrm{KN})$ or tracer. The $\mathrm{KN}$ is a nuclide which is systematically measured in each single waste package, with a relative long half-life (years or dozens of years) and whose activity can be correlated to the activity of DTM and ITM radionuclides.

When the radionuclide inventory is available, we collect a repre-

\footnotetext{
* Correspondence to: CERN, Route de Meyrin, CH-1211, Geneva 23, Switzerland.

E-mail address: biagio.zaffora@cern.ch (B. Zaffora).
} 


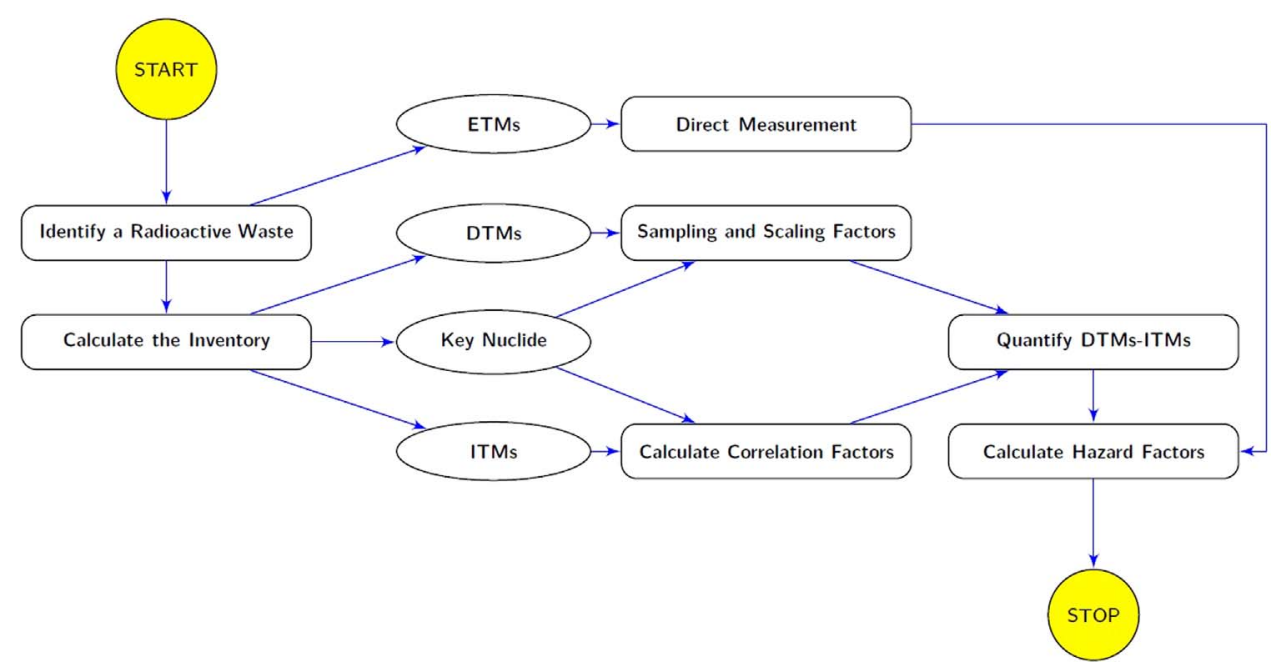

Fig. 1. Radiological characterization process adopted at CERN for very-low-level radioactive waste.

sentative sample to evaluate the activity of the DTMs using the so-called Scaling Factor method (ISO, IAEA, 2009) and perform calculations to estimate the activity of ITMs using the Correlation Factor method (ISO, ISO 16966, 2013.).

We finally measure each single waste package via $\gamma$-ray spectrometry and use the activity of the KN to estimate the activity of DTMs and ITMs. The activities so obtained are compared to the limits given by national authorities for waste management to check for the acceptability of the waste in the disposal facilities. Fig. 1 shows a diagram illustrating the principal stages of the process.

Section 2 describes the stages of the radiological characterization process including the methods to quantify ETM, DTM and ITM radionuclides and the waste acceptability criteria. Section 3 presents the results of the calculation of the radionuclide inventories for representative materials activated at CERN together with the application of the characterization method to a metallic waste population. Section 4 discusses the results obtained and the production mechanisms of the main radionuclides produced by activation of metals. Conclusions are given in the last section.

\section{Methods}

\subsection{Identification of waste and calculation of radionuclide inventories}

At CERN, a waste elimination campaign starts with the identification of a waste batch. A batch is often chosen based on the volume it occupies in the temporary storage area. When the perimeter of a waste population is defined we start collecting information about its radiological history and chemical composition. Based on this information we calculate the radionuclide inventory using the analytical code Actiwiz (Theis and Vincke, 2012.), which relies on extensive Monte Carlo simulations carried out using Fluka (Battistoni, , 2006; Ferrari, 2005).

When dealing with legacy waste we often have limited knowledge of its radiological history. To predict the complete list of possible radionuclides generated by activation we need to consider a large number of potential scenarios. A scenario is described as the combination of a beam energy, a position inside the accelerator tunnel, an irradiation and a decay time. For the present study we considered beam energies from $160 \mathrm{MeV}$ (Linac 4) up to $7 \mathrm{TeV}$ (Large Hadron Collider), covering the energy spectrum of CERN's proton accelerators. The locations inside the accelerator tunnels span from the beam impact area to the zones behind the concrete shielding. Irradiation and decay times range from a few months to 30 years.

To establish a representative inventory of radionuclides from activated metals we also considered a list of 43 chemical compositions from CERN's materials catalogue (Froeschl and alter, 2012.). We grouped these compounds into 3 families as follows:

- steel, including 16 grades, with density in the range $7-8.75 \mathrm{~g} / \mathrm{cm}^{3}$;

- aluminium, including 7 grades, with density in the range 2.66$2.84 \mathrm{~g} / \mathrm{cm}^{3}$;

- copper, including 20 grades, with density in the range 7.6-8.94 g/ $\mathrm{cm}^{3}$.

For the calculations we used the average value of the concentration of each element, as given by reference standards. The variability of a given element's content is taken into account via the dispersion between the various grades. Table 1 shows a short list of the major elements for common material types used at CERN.

\subsection{Quantification of the activity in waste packages}

\subsubsection{Measurement of easy-to-measure radionuclides}

The quantification of ETM radionuclides, including the Key Nuclide, is made via $\gamma$-ray spectrometry. This technique is well known and detailed information can be found in numerous classical references (Knoll, 2010; Gilmore, 2008). At CERN, various in-situ and fixed germanium detectors are used for the measurement of $\gamma$-emitters in waste packages and samples. The detectors are either nitrogen or electrically cooled and their efficiency for Co-60 at $1.33 \mathrm{MeV}$ spans between $30 \%$ and $60 \%$.

We performed a sensitivity study to take into account the variability of various physical parameters such as the apparent density of a waste package, the distance package-detector and the height of the waste within a package. Standard efficiency calibration functions were then created and used to estimate the uncertainty associated with the

Table 1

List of major elements for 4 reference chemical compositions used at CERN. Values in parenthesis are given as weight fractions.

\begin{tabular}{ll}
\hline Material grade & Chemical elements and reference concentrations \\
\hline Aluminium 6060 & $\mathrm{Al}(98.375), \mathrm{Cr}(0.05), \mathrm{Cu}(0.1), \mathrm{Fe}(0.2), \mathrm{Mg}(0.475)$, \\
& $\mathrm{Mn}(0.1), \mathrm{Si}(0.45), \mathrm{Ti}(0.1), \mathrm{Zn}(0.15)$ \\
Steel 304L & $\mathrm{C}(0.03), \mathrm{Cr}(18.5), \mathrm{Co}(0.1), \mathrm{Fe}(67.0825), \mathrm{Mn}(2.0)$, \\
& $\mathrm{Ni}(11.25), \mathrm{P}(0.0225), \mathrm{Si}(1.0), \mathrm{S}(0.015)$ \\
Copper OFE & $\mathrm{Bi}(0.001), \mathrm{Cd}(0.0001), \mathrm{Cu}(99.99), \mathrm{Pb}(0.001)$, \\
& $\mathrm{Hg}(0.0001), \mathrm{O}(0.0005), \mathrm{S}(0.0018), \mathrm{Zn}(0.0001)$ \\
Low carbon content & $\mathrm{C}(0.105), \mathrm{Fe}(99.35), \mathrm{Mn}(0.45), \mathrm{P}(0.02), \mathrm{S}(0.025)$, \\
steel & $\mathrm{Si}(0.05)$ \\
\hline
\end{tabular}


Table 2

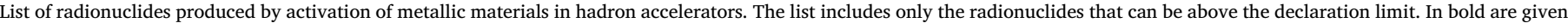
the identified Key Nuclides.

\begin{tabular}{|c|c|c|}
\hline Material & Easy-to-measure nuclides & Difficult- and impossible-to-measure nuclides \\
\hline Aluminium & Al-26, Na-22, Ti-44, Mn-54, Co-57, Co-60, Zn-65 & H-3, Be-10, C-14, Cl-36, Ar-39, Ca-41, Fe-55, Ni-63 \\
\hline Steel & Na-22, Ti-44, Mn-54, Co-57, Co-60, Zn-65, Ge-68, Nb-94, Ag-108 m & $\begin{array}{l}\text { H-3, C-14, Cl-36, Ar-39, Ca-41, V-49, Fe-55, Ni-63, Nb-91, Mo-93, } \\
\text { Nb-9 m, Tc-99 }\end{array}$ \\
\hline Copper & $\begin{array}{l}\text { Na-22, Al-26, Ti-44, Mn-54, Co-57, Co-60, Zn-65, Rh-101, Rh-102, Ag-108 m, Ag-110m, } \\
\text { Sn-121 m, Sb-125, Ba-133, Hf-172, Hg-194, Pb-202, Bi-207 }\end{array}$ & $\begin{array}{l}\text { H-3, C-14, Cl-36, Ca-41, Ar-39, V-49, Fe-55, Ni-63, Sr-90, Mo-93, } \\
\text { Sn-119 m, Gd-148, Pt-193 }\end{array}$ \\
\hline
\end{tabular}

efficiency calibration function.

Potential heterogeneities of the activity distribution inside a waste package are taken into account by averaging the upper bounds (confidence interval at 95\%) of multiple measurements on different sides of the waste. Moreover, the use of upper bounds of confidence intervals minimizes the risk associated with the presence of activity concentration. Activity concentrations are also controlled in the sorting process when a limit in terms of dose rate is fixed for each single item of waste.

The counting time is set in order to allow the detection of activity levels below the declaration limits of the ETMs (see Section 3.1, Table 2) and to minimize the uncertainty on the peak area at $95 \%$ confidence level.

\subsubsection{Scaling factors and quantification of Difficult-to-Measure radionuclides}

A scaling factor is a parameter used to calculate the activity of Difficult-to-Measure nuclides from that of a Key Nuclide, when an experimental correlation exists between DTM and KN (ISO, "ISO 21238, 2007; IAEA, 2009) To check for correlations, we collect a representative sample from the waste population and measure the activities of both DTM and KN. We calculate the coefficient of determination $R^{2}$ and, based on this value, we estimate the Scaling Factor. In particular, if $R^{2} \geq 0.7$, we can use linear regression to estimate the activity $a_{D T M}$ of a DTM nuclide from the activity $a_{K N}$ of the KN.

In case of strong positive correlation, an alternative to the linear regression consists of checking the underlying distribution of the experimental ratios $S F_{i}=a_{D T M, i} / a_{K N, i}$ and calculating the best average content estimator for the distribution. In practice, the distribution of the ratios is often log-normal and the geometric mean is used to calculate a representative average scaling factor (IAEA, 2009).

Whether the SF is calculated using the linear model or the geometric mean, we predict the activity of the DTM radionuclide $\hat{a}_{D T M, j}$ in the package $\mathrm{j}$ as:

$\hat{a}_{D T M, j}=S F \times a_{K N, j}$

where SF is either the slope of the linear model or the geometric SF and $a_{K N, j}$ is the measured activity of the $\mathrm{KN}$ (in $\mathrm{Bq} / \mathrm{g}$ ) in the package $\mathrm{j}$.

The uncertainty of SF is calculated differently based on the methodology used to estimate the scaling factor. If linear models are applied, the uncertainty of the slope is calculated as the standard error of the slope (Hastie et al., 2009). If we use the geometric SF, the dispersion $D$ around the average content estimator is used.

If $R^{2}<0.7$ the arithmetic mean or the median of the measured activities of the DTMs can be used to estimate the concentration of the DTMs in each package: this is called Mean Activity Method. Following the skewness of the underlying data, one of these two average estimators must be preferred to the other for conservativeness. For right-skewed data the mean is to be preferred while the median will be preferred for left-skewed distributions. Standard errors of the mean or interquartile ranges are used to estimate the uncertainty of the DTMs mean activity.

A detailed description of these techniques can be found in reference (Zaffora et al., 2016).
2.2.3. Correlation method and quantification of impossible-to-measure radionuclides

The Correlation Method is one of the so-called "Range Methods" described in reference (ISO, "ISO 16966, 2013.). It consists of identifying a representative number of potential activation scenarios for a batch of waste and calculating the correlation between a Key Nuclide and the DTM and ITM nuclides for each one of these scenarios.

In practice, correlation factors (CF) are similar to Scaling Factors but, instead of being calculated from measurements on samples, they are estimated via analytical calculations or Monte Carlo simulations. For the radiological characterization of very-low-level activity waste produced at CERN we use the Correlation Method to estimate the activity of Impossible-to-Measure radionuclides and also to predict ranges for the scaling factors.

The specific activity $\hat{a}_{I T M, j}$ of an ITM nuclide in package $j$ is estimated as follows:

$\hat{a}_{I T M, j}=C F \times a_{K N, j}$

where $a_{K N, j}$ is the measured specific activity (in $\mathrm{Bq} / \mathrm{g}$ ) of the Key Nuclide in the package $j$ and $C F$ is the calculated correlation factor. As for the scaling factors, we can use various methods to quantify the best estimator of CF. A common procedure consists in studying the underlying distribution of $\mathrm{CFs}$ and choosing among mean, median or geometric mean correlation factor according to this distribution.

To estimate the uncertainty of CF we use both classical and statistical learning methods. In particular we considered multiple linear regression, decision trees, bootstrap aggregation and random forests to estimate prediction errors of CFs. A detailed study of these methodologies is behind the purpose of the present study and their description can be found in reference (Hastie et al., 2009).

\subsection{Waste acceptability and hazard factors}

The French National Agency for Radioactive Waste Management (ANDRA) defined a hazard factor, called IRAS (French acronym: Indice Radiologique d'Acceptation en Stockage), to establish criteria for waste acceptability in disposal facilities (ANDRA, 2013.). The IRAS of a waste package is defined as:

$\operatorname{IRAS}=\sum_{i} \frac{a_{i}}{A L_{i}}$

where $a_{i}$ is the specific activity (in $\mathrm{Bq} / \mathrm{g}$ ) of the radionuclide $i$ within the package and $A L_{i}$ is the activity limit of the radionuclide $i$. For a batch of waste to be accepted at the TFA storage centre the average IRAS of all the waste packages needs to be below 1 and the IRAS of each single package must be below 10 .

If the activity of a radionuclide is above the declaration limit DL fixed by ANDRA, its activity must be considered for the calculation of the IRAS. When a large number of activation scenarios are taken into account, we must check if for each single scenario a given radionuclide can be above its DL. To do so we calculate the normalized specific activity $a_{i}^{I R A S=10}$ of the radionuclide $i$ for a given scenario when IRAS $=10$ as follows: 
$a_{i}^{I R A S=10}=\frac{a_{i}}{\sum_{j} \frac{a_{j}}{A L_{j}}} \times 10$

where $a_{i}$ is the specific activity of the radionuclide $i$ (in Bq/g/primary/ s) and the summation represents the total IRAS for a beam loss of 1 particle per second. Eq. (4) gives the specific activity of radionuclide $i$ when the IRAS is 10 . We then compare $a_{i}^{I R A S=10}$ with the declaration limit $D L$ of the radionuclide $i$ and include the radionuclide in the inventory if:

$a_{i}^{\text {IRAS }=10}>D L_{i}$.

For practical reasons we discriminate between DTM and ITM radionuclides depending on their contribution to the IRAS. In particular, we define the contribution $C_{i}$ of a radionuclide $i$ to the IRAS as:

$C_{i}=\frac{\frac{a_{i}}{A L_{i}}}{\sum_{j} \frac{a_{j}}{A L_{j}}}$.

If a radionuclide contributes to more than $1 \%$ of the IRAS we measure it (direct ETM measurement and Scaling Factors for DTMs) otherwise it is estimated using the Correlation Method (ITMs).

\section{Results}

\subsection{Radionuclide inventory of activated metals}

Table 2 presents the complete list of ETM, DTM and ITM radionuclides produced via activation of representative metallic materials at CERN (i.e. aluminium, steel and copper), using an extensive set of possible chemical compositions taken from CERN's material catalogue. This list was obtained performing the calculations described in Section 2.1. A radionuclide is included in Table 2 if it respects the condition of Eq. (5). Radionuclides in bold are the identified Key Nuclides.

The radionuclide inventory of Table 2 represents the list of expected nuclides. The presence of impurities and traces in a metal can potentially induce the production of radionuclides that are not included in this list. In particular, when a special alloy is used dedicated studies should be carried out to estimate the radionuclide inventory. Common material types (such as Copper OFE, ETP and DHP or Aluminium 6060, 6061 and 6082) can however be considered as similar from a radiological point of view because they produce similar radionuclide inventories.

For the actual calculation of Correlation Factors and of the contributions to the IRAS, according to Eq. (6), we restricted our analysis to the most representative chemical compositions of aluminium, steel and copper used at CERN. The results are given in Tables 36. Only the radionuclides contributing more than $0.1 \%$ to the IRAS are shown. We calculated the Correlation Factor as the geometric mean of the CFs over 630 activation scenarios as described in Section 2.1.

In Section 4 we will discuss the production mechanisms of the most important radionuclides given in Tables 3-6.

\subsection{Validation}

The radiological characterization process that we introduced in this

Table 3

Correlation factors (CF) and contribution to the IRAS of radionuclides from activated aluminium 6060. Na-22 is the identified Key Nuclide.

\begin{tabular}{lll}
\hline Nuclide & Correlation factor & Contribution to IRAS \\
\hline H-3 & 11 & $9.46 \%$ \\
Na-22 & 1 & $89.80 \%$ \\
Ti-44 & $1.4 \times 10^{-3}$ & $0.13 \%$ \\
Co-60 & $5.2 \times 10^{-3}$ & $0.47 \%$ \\
Total & & $99.86 \%$ \\
\hline
\end{tabular}

Table 4

Correlation factors and contribution to the IRAS of radionuclides from activated steel 304L. Co-60 and Ti-44 are the identified Key Nuclides.

\begin{tabular}{llll}
\hline Nuclide & $\begin{array}{l}\text { Correlation factor } \\
\text { using Co-60 as KN }\end{array}$ & $\begin{array}{l}\text { Correlation factor } \\
\text { using Ti-44 as KN }\end{array}$ & $\begin{array}{l}\text { Contribution to } \\
\text { IRAS }\end{array}$ \\
\hline $\mathrm{H}-3$ & 1.4 & 53 & $1.28 \%$ \\
$\mathrm{Na}-22$ & $4.1 \times 10^{-3}$ & 0.15 & $0.37 \%$ \\
$\mathrm{Ar}-39$ & 0.4 & 15 & $0.37 \%$ \\
$\mathrm{Ti}-44$ & 0.03 & 1 & $2.43 \%$ \\
$\mathrm{Fe}-55$ & 4.6 & 170 & $4.15 \%$ \\
Co-60 & 1 & 38 & $91.33 \%$ \\
Total & & & $99.93 \%$ \\
\hline
\end{tabular}

Table 5

Correlation factors and contribution to the IRAS of radionuclides from activated copper CuOFE. Co-60 is the identified Key Nuclide.

\begin{tabular}{lll}
\hline Nuclide & Correlation factor & Contribution to IRAS \\
\hline $\mathrm{H}-3$ & 1.7 & $1.60 \%$ \\
$\mathrm{Ti}-44$ & $1.2 \times 10^{-3}$ & $0.12 \%$ \\
$\mathrm{Fe}-55$ & 0.2 & $0.19 \%$ \\
$\mathrm{Co}-60$ & 1 & $96.57 \%$ \\
$\mathrm{Ni}-63$ & 1.6 & $1.50 \%$ \\
Total & & $99.98 \%$ \\
\hline
\end{tabular}

Table 6

Correlation factors and contribution to the IRAS of radionuclides from activated steel low carbon content. Ti-44 is the identified Key Nuclide.

\begin{tabular}{lll}
\hline Nuclide & Correlation factor & Contribution to IRAS \\
\hline $\mathrm{H}-3$ & 84 & $15.84 \%$ \\
$\mathrm{Na}-22$ & 0.05 & $0.89 \%$ \\
Ti-44 & 1 & $18.88 \%$ \\
Mn-54 & 0.05 & $1.00 \%$ \\
Fe-55 & 340 & $63.34 \%$ \\
Total & & $99.95 \%$ \\
\hline
\end{tabular}

Table 7

Materials and weights for 4 disposal campaigns of TFA waste.

\begin{tabular}{lllll}
\hline Campaign ID & $\begin{array}{l}\text { Aluminium } \\
\text { (tons) }\end{array}$ & $\begin{array}{l}\text { Copper } \\
\text { (tons) }\end{array}$ & $\begin{array}{l}\text { Steel } \\
\text { (tons) }\end{array}$ & $\begin{array}{l}\text { Total weight per } \\
\text { campaign (tons) }\end{array}$ \\
\hline 1 & 5.2 & - & 36.8 & 42 \\
2 & 7.1 & - & 32.3 & 39.4 \\
3 & 42.2 & - & 81 & 123.2 \\
4 & 12.6 & 38.7 & 168.6 & 219.9 \\
Total weight (tons) & & & 424.5 \\
\hline
\end{tabular}

paper is presently used at CERN in various elimination campaigns. As an example, we describe the results of 4 campaigns consisting of legacy activated metals sent to the French TFA disposal facility in 2015 and 2016. Table 7 shows the weight of each material in the 4 waste populations.

The TFA waste was packaged into containers with an internal volume of $1.35 \mathrm{~m}^{3}$. The 4 campaigns consisted of 53 waste packages filled with aluminium, 17 with copper and 140 with steel, where by "steel" we mean both low-carbon-content and $304 \mathrm{~L}$ types. Aluminium represents $16 \%$ of the total weight, Copper $9 \%$ and steel $75 \%$.

The waste of the 4 campaigns is legacy metallic material activated in proton accelerators. Their exact position inside the accelerators is unknown, along with the irradiation and decay times. The waste batches consist of pipes, cables supports and metals used to build the accelerating systems.

The complete list of radionuclides potentially above the Declaration Limits is given in Tables 2-5. From the tables we can see that the DTM nuclides are $\mathrm{H}-3$ in aluminium, $\mathrm{H}-3$ and Fe-55 in steel and $\mathrm{H}-3$ and Ni- 
Table 8

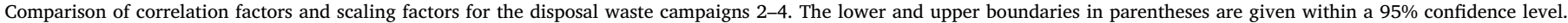

\begin{tabular}{|c|c|c|c|c|c|c|}
\hline Material & KN & DTM & $\mathrm{CF}$ & SF Camp. 2 & SF Camp. 3 & SF Camp. 4 \\
\hline Aluminium & $\mathrm{Na}-22$ & $\mathrm{H}-3$ & $11(0.07,1500)$ & $4.4(0.3,65)$ & $1500(66,34100)$ & $71(9,570)$ \\
\hline \multirow[t]{2}{*}{ Copper } & Co- 60 & $\mathrm{H}-3$ & $1.7(0.1,22)$ & - & - & $15(0.9,250)$ \\
\hline & & $\mathrm{Ni}-63$ & $1.6(0.07,34)$ & $8.1(1.3,52)$ & - & $9.2(0.5,190)$ \\
\hline Steel & $\mathrm{Ti}-44$ & $\mathrm{H}-3$ & $84(4,1700)$ & $33(0.3,3350)$ & - & $71(9,530)$ \\
\hline
\end{tabular}

63 in copper: these radionuclides are quantified using the SF method if $\mathrm{R}^{2}$ is above 0.7 . We collected 219 samples from the 4 campaigns and we performed 347 radiochemical analysis to estimate the DTM radionuclides. In average, 1 sample per $1.94 \mathrm{t}$ of waste is collected. This corresponds to the measurement of 1 DTM radionuclide via radiochemical analysis per $1.22 \mathrm{t}$ of waste. Table 8 gives the geometric SFs (see (Zaffora et al., 2016)) obtained from the sampling campaigns. Calculated $\mathrm{CFs}$ and confidence intervals at $95 \%$ are given for comparison. A discussion of these results will be presented in Section 4.

The activity of H-3 in the aluminium samples of campaign 1 is below the detection limit. For its quantification we used the Mean Activity Method which gives an activity equal to $0.75 \mathrm{~Bq} / \mathrm{g}$. No activated copper was present and samples of this material were not collected. The activity levels of both H-3 and Fe-55 in steel are also consistently below the detection limits. Their activities are estimated using the Mean Activity Method. The average values of H-3 and Fe-55 are equal to $0.07 \mathrm{~Bq} / \mathrm{g}$ and $2.2 \mathrm{~Bq} / \mathrm{g}$ respectively. We could not use SFs to estimate DTMs for campaign 1 and therefore they are not included in Table 8.

In campaign 2, except for the pair $\mathrm{H}-3 / \mathrm{Ti}-44$, no correlations were found in steel waste for both $\mathrm{H}-3$ and Fe-55 with the Key Nuclides. The amount of Fe-55 in the waste packages is estimated using the Mean Activity Method and is equal to $43.96 \mathrm{~Bq} / \mathrm{g}$. For operational reasons the $\mathrm{H}-3$ was not measured in copper and its concentration was estimated via calculations. The pairs $\mathrm{H}-3 / \mathrm{Na}-22$ in aluminium and $\mathrm{Ni}-63 / \mathrm{Co}-60$ in copper show a strong positive correlation and the SFs are calculated.

The activities of $\mathrm{H}-3$ and Fe-55 on the collected samples of steel for campaign 3 are very low and often below the detection limits. The estimated mean activities of $\mathrm{H}-3$ and Fe-55 are $0.04 \mathrm{~Bq} / \mathrm{g}$ and $6.54 \mathrm{~Bq} / \mathrm{g}$ respectively. No copper waste is present. The experimental SF for the pair $\mathrm{H}-3 / \mathrm{Na}-22$ is calculated for aluminium.

Finally, for campaign 4, the SF are used to estimate the activities of H-3 and Fe-55 from Ti-44 in steel. No experimental correlation was found between the 2 DTMs and the Key Nuclide Co-60. The SFs are also used to estimate the DTMs in aluminium and copper.

Fig. 2 shows the distributions of the specific activity of major ETM and DTM radionuclides for the 4 campaigns considered. The DTM activities are either calculated using the Mean Activity Method or the SFs of Table 8 in combination with Eq. (1). The plots are given per material type. The mean activity value is also shown. We are in presence of right-skewed distributions with a moderate amount of potential outliers.

The activity of the ITM radionuclides is obtained by multiplying the correlation factors (see Tables 2-5) and the measured activity of the Key Nuclide in each waste package. If multiple Key Nuclides can be used, the KN having the highest statistical correlation with the ITM is chosen. Among the calculated ITM, no radionuclides exceeding the Declaration Limits were found. They are excluded from the calculation of the hazard factors.

\section{Discussion}

\subsection{Analysis of the characterization process}

Analytical calculations are used to establish the list of important radionuclides, to estimate the range of $\mathrm{SFs}$ and to estimate $\mathrm{CFs}$. In Table 8 we compared the calculated correlation factors and the measured scaling factors for the DTMs of the waste campaigns
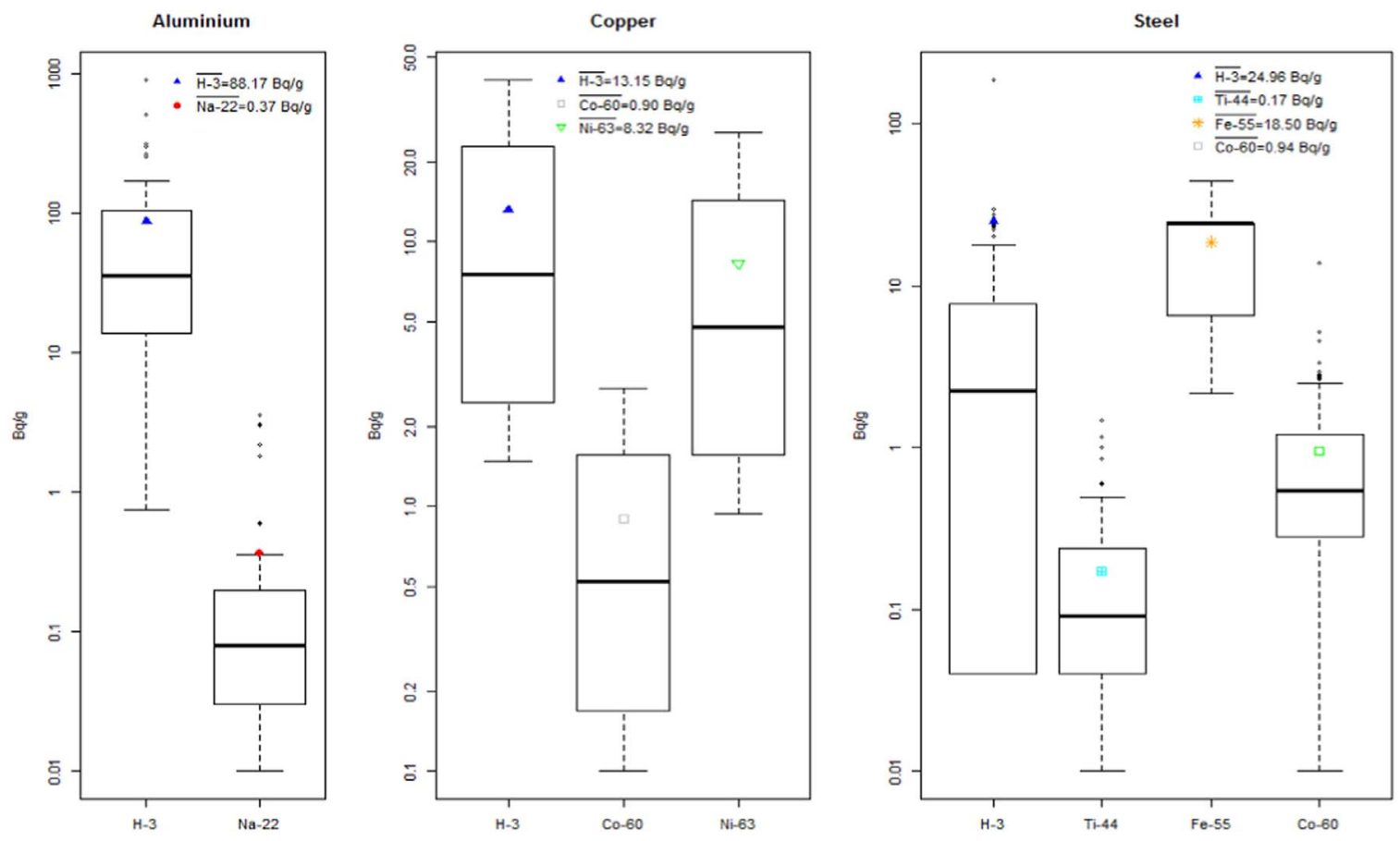

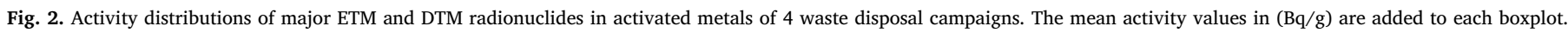


considered. We can see that the confidence intervals of CFs systematically contain experimental SFs with the exception of the scaling factor of $\mathrm{H}-3$ in aluminium for campaign 3. In this case the experimental SF is very close to the upper bound of the $\mathrm{CF}$.

The calculated geometric CF is in general a good estimator of the geometric SF and CFs predict scaling factors within 1 order of magnitude. The error associated to the difference between the CFs and the SFs is negligible because we use only experimental values to estimate DTMs and because the level of activity of ITM radionuclides is well below the Declaration Limit.

We collected complementary information to investigate the discrepancy between the $\mathrm{CF}$ and the SF of the pair $\mathrm{H}-3 / \mathrm{Na}-22$ in the aluminium of campaign 3 . We found that the waste was stored for at least 20 years in CERN's storage. We performed a new set of calculations fixing all the input parameters given in Section 2.1 with the exception of the decay time that now spans from 20 up to 40 years, with steps of 5 years. The new calculation includes 1050 scenarios. The geometric CF obtained is 280 and the confidence interval is $(11,6900)$. The amount of Na-22 $\left(\mathrm{T}_{1 / 2}=2.6\right.$ years $)$ decreases faster than $\mathrm{H}-3$ $\left(\mathrm{T}_{1 / 2}=12.3\right.$ years $)$ and for long decay times the ratio of activities $\mathrm{H}$ $3 / \mathrm{Na}-22$ increases. The confidence interval now includes the experimental SF and the calculated $\mathrm{CF}$ is within 1 order of magnitude of the SF. Another set of calculation was performed including only decay times longer or equal to 30 years. The new $\mathrm{CF}$ is 800 and the confidence interval $(99,6500)$. As expected, the value of $\mathrm{CF}$ increases again.

In the previous example we showed that calculations and experiments are in agreement. For historical waste the radiological history of the waste is often unknown and large scale calculations can provide a robust estimation of Scaling Factors. We also showed how new input data can be used to modify the analytical calculations in order to adapt the predictive model to experimental results.

\subsection{Calculations and production mechanisms of principal radionuclides}

The production mechanisms of the nuclides of interest for radioactive waste characterization are complex and their detailed treatment can be found in classical references such as in (Barbier, 1969). We shortly describe here the principal production mechanisms of the nuclides obtained from calculations (Tables 3-6), experimentally quantified and contributing for more than $1 \%$ to the IRAS.

The key-nuclides of aluminium 6060, Na-22, and the DTM H-3 are mainly produced via spallation of hadrons with the chemical element Al. A small amount of both nuclides is also produced via spallation from $\mathrm{Mg}$ and $\mathrm{Si}$. Na-22 is produced in small quantity via nuclear reactions with $\mathrm{Mg}$ such as $\mathrm{Mg}^{25}$ (p, $\left.\alpha\right) \mathrm{Na}^{22}$ (Browne, 1959).

In steel $304 \mathrm{~L}$, the radionuclide $\mathrm{H}-3$ is typically produced from spallation and nuclear interaction with $\mathrm{Fe}$ and Cr. Small amounts of tritium are produced via spallation with $\mathrm{Mn}, \mathrm{Ni}$ and $\mathrm{Si}$. Fe-55 is produced via spallation and nuclear interactions with $\mathrm{Fe}$. Common production mechanisms of $\mathrm{Fe}-55$ are $\mathrm{Fe}^{54}(\mathrm{n}, \gamma) \mathrm{Fe}^{55}$ and $\mathrm{Fe}^{56}(\mathrm{n}, 2 \mathrm{n}) \mathrm{Fe}^{55}$ (Audi et al., 2003). Ti-44 is mainly produced from spallation with $\mathrm{Cr}$, $\mathrm{Mn}$ and Ni. The Key-Nuclide Co-60 is produced via neutron capture with Co-59.

All the radionuclides of interest produced in copper are mainly due to spallation and nuclear interactions with the element $\mathrm{Cu}$ itself, which represents over $99.9 \%$ of the total weight. The presence of the element Co as impurity can significantly increase the amount of Co-60 produced.

The chemical element Fe has a very high weight fraction in the material type steel low carbon content (i.e., 99.35\%), which explains why most of the radionuclides in this metal are produced via nuclear interactions with Fe. A small fraction of Mn-54 is also produced via nuclear interactions with natural occurring $\mathrm{Mn}$.

\section{Conclusion}

The characterization process described in the present article is based on analytical calculations and measurement of important radionuclides. The calculations presented allow us to predict the radionuclide inventory for common CERN activation scenarios. The ETM radionuclides are systematically measured and the DTMs are estimated using either the Scaling Factor or the Mean Activity Methods. ITM radionuclides have a negligible impact on hazard factors calculated from very-low-level metallic waste. The treatment, analysis and final disposal of more than $420 \mathrm{t}$ of metallic waste have demonstrated the robustness of the characterization process.

The large number of samples collected and the repeated and consistent prediction of experimental scaling factors via the calculation of correlation factors demonstrates the applicability of the method at CERN. The process can also be applied to other facilities where the activation mechanisms are similar to the ones of the European Organization for Nuclear Research.

The radiological history of legacy waste is often unknown. To remediate this lack of information we consider a large number of irradiation scenarios that allow us to estimate average correlation factors between $\gamma$-emitters and Difficult- or Impossible-to-Measure radionuclides. The measure of ETMs in each waste package and the use of scaling factors, correlation factors and the Mean Activity Method enable us to quantify the specific activity of each produced radionuclide in a batch of waste.

We demonstrated that when a CF differs from the experimental SF a new set of calculations can be generated in order to take into account complementary information from experimental data and improve the predictive power of the model. The decay time plays an important role especially when the half-life of Key Nuclide and DTM is very different, such as for the pair Na-22 and H-3 in aluminium.

The characterization process here described is presently used for metals and cables, and it is foreseen to extend its application to other materials including concrete and plastic. The method is robust with respect to uncertainties for possible activation scenarios (which is the typical case of legacy waste), because it uses statistical techniques and experimental data to reduce the impact of such uncertainties on the radiological characterization.

\section{Acknowledgement}

The authors wish to thank Luca Bruno, Francesco Paolo La Torre, Yvon Algoet, Bertrand Cellerier, Alexandra Nagy and Nick Walter for their contributions to the calculations, the sampling and all the aspects related to the management of the disposal campaigns.

\section{References}

ANDRA, 2013. Critères radiologiques d'acceptation des déchets TFA, SUR.SP.AMES.02. 0007/D, Chatenay-Malabry (France).

Audi, G., Bersillon, O., Blachot, J., Wapstra, A., 2003. "The Nubase evaluation of nuclear and decay properties". Nuclear Physics A, vol. 729 (1), 3-128.

Barbier, M.M., 1969. Induced Radioactivity. North-Holland, Amsterdam.

Battistoni, G., 2006. The FLUKA code: Description and benchmarking. In: Proceedings of the Hadronic Shower Simulation Workshop, Fermilab, 2006

Browne, C.P., 1959. "Energy levels of Na22 from the Mg24(d, a)Na22 and Mg25(p, $\alpha$ ) Na22 reactions". Nuclear Physics, vol. 12 (6), 662-671.

Ferrari, A., 2005. FLUKA: a multi-particle transport code. CERN-2005-10 Geneva.

Froeschl, R., Theis, C., La Torre, F., Vincke, H., Walter, N., 2012. Radiological hazard classification of materials in CERN's accelerators, CERN-DGS-2012-003-RP-IR, EDMS ID: 1184236 , Geneva.

Gilmore, G., 2008. Practical Gamma-ray Spectrometry. John Wiley \& Sons, Inc, Chichester, West Sussex, England.

Hastie, T., Tibshirani, R., Friedman, J., 2009. The Elements of Statistical Learning: Data Mining Inference and Prediction. Springer-Verlag, Stanford, California.

IAEA, 2007. Strategy and methodology for radioactive waste characterization, Vienna: IAEA-TECDOC-1537. 
IAEA, 2009. No. NW-T-1.18. Determination and use of scaling factors for waste characterization in nuclear power plants. IAEA, Vienna.

ISO, "ISO 16966, 2013. Nuclear energy - Nuclear fuel technology - Theoretical activation calculation method to evaluate the radioactivity of activated waste generated at nuclear reactors. International Standard Organization, Geneva.

ISO, "ISO 21238, 2007. Nuclear energy - Nuclear fuel technology - Scaling factor method to determine the radioactivity of low- and intermediate-level radioactive waste packages generated at nuclear power plants," International Standard Organization, Geneva.
Knoll, G.F., 2010. Radiation Detection and Measurement. John Wiley \& Sons, Inc, Hoboken, NJ, USA.

Theis, C., Vincke, H., 2012. Actiwiz - Optimizing your nuclide inventory at proton accelerators with a computer code. In: Proceedings of the ICRS12 conference, Nara (Japan).

Zaffora, B., Magistris, M., Saporta, G., La Torre, F.P., 2016. Statistical sampling applied to the radiological characterization of historical waste. Eur. Phys. J. Nucl. Sci. Technol. 2 (34). 